\title{
Évolution du cancer du testicule en France
}

\author{
Guy HEDELIN ${ }^{*}$, Laurent REMONTET ${ }^{* *}$
}

*Registre des cancers du Bas-Rhin, Université Louis Pasteur Strasbourg I, Faculté de médecine, Strasbourg.
**aboratoire de biostatistique, Université Claude-Bernard Lyon I. Réseau Francim

\section{RESUME}

Comme dans beaucoup de pays, en France la mortalité par cancer du testicule a diminué, passant de 0,75 en 1978 à 0,25 pour 100000 en 2000 (taux standardisé sur la population mondiale).

L'incidence a augmenté sur la même période, passant de 3,17 en 1978 à 4,82 pour 100000 en 2000 (taux standardisé sur la population mondiale). Cependant si les séminomes n'ont cessé d'augmenter pour toutes les cohortes de naissance, l'incidence des non séminomes a diminué pour les cohortes de naissance entre les deux guerres mondiales, avant d'augmenter parallèlement aux séminomes. Ce comportement est pour l'instant inexpliqué, mais ne semble pas être un artefact.

II existe un gradient nord/sud et ouest/est, les taux d'incidence allant de 4,0 dans le sud-ouest à $\mathbf{8 , 0}$ dans le nord-est.

On estime actuellement, en France, à 1500 le nombre de nouveaux cas de cancers du testicule chaque année, dont 960 chez les hommes de 15 à 40 ans.

Mots clés : cancer du testicule, incidence, mortalité, évolution, épidémiologie

\section{INTRODUCTION}

Le cancer du testicule est un cancer rare et de bon pronostic. Malgré son comportement spécifique, il reste préoccupant à plusieurs titres : son évolution au cours du temps montre généralement une augmentation $[1,2,5,10]$, il touche principalement des hommes jeunes pour lesquels les conséquences psychologiques sont importantes en particulier en ce qui concerne la possibilité d'avoir ultérieurement des enfants, enfin c'est le cancer le plus fréquent chez l'homme jeune.

En France les données sont relativement rares $[6,7,9]$ et aucune étude n'a encore été menée sur l'évolution temporelle au niveau national. Les registres de cancer ont maintenant un recul suffisant (entre 10 et 25 ans pour 9 d'entre eux) pour analyser les tendances chronologiques et faire des estimations pour la France entière.

\section{MATERIEL ET METHODE}

Les données d'incidence proviennent des 9 registres généraux suivants (entre parenthèses est donnée la première année d'enregistrement) : Calvados (1978), Doubs (1976), Hérault (1983), Isère (1978), Manche (1994), Bas-Rhin (1975), Haut-Rhin (1989), Somme (1982) et Tarn (1981). Les données d'évolution seront parfois présentées en séparant les registres "anciens" (ceux ayant débuté dans les années 70) et les registres dits "nouveaux". La période utilisée pour chaque registre va de la première année d'enregistrement jusqu’à 1997.

\footnotetext{
Correspondance :

Dr Guy Hédelin - Laboratoire d'épidémiologie et de santé publique, Registre des cancers du Bas-Rhin, Faculté de médecine, 67085 Strasbourg cedex - Tel 0390243198 Fax 0390243189 -

Email Guy.Hedelin@medecine.u-strasbg.fr
} 
Les données de mortalité proviennent du Service commun 8 de l'INSERM. Seule la notion de cancer du testicule est notifiée, il n'est pas possible de séparer les séminomes des non séminomes.

Les extrapolations à l'an 2000 ou à certaines cohortes de naissance reposent sur un modèle âge-période-cohorte [4]. Les estimations sur la France entière utilisent le rapport entre la mortalité et l'incidence. Ce rapport est constant au cours de la période considérée dans les régions couvertes par les registres et l'hypothèse est faite qu'il en est de même dans les autres régions [3].

Comme la mortalité n'est pas connue par type histologique, la répartition entre séminomes et non séminomes dans les régions couvertes par les registres est utilisée pour l'extrapolation au niveau national.

Les risques relatifs (RR) sont tous exprimés par rapport à la cohorte de naissance de 1968.

\section{RESULTATS}

\section{1. Évolution sur la période 1978 - 2000.}

Le taux de mortalité, standardisé sur le monde, est passé de 0,75 en 1978 à 0,25 pour 100000 en 2000 , soit une diminution de 4,9\% par an (Figure 1).

Le taux d'incidence, standardisé sur le monde, de l'ensemble des cancers du testicule est passé de 3,17 en 1978 à 4,82 pour 100000 en 2000 , soit une augmentation de $1,9 \%$ par an (Figure 2).

L'augmentation des séminomes est identique dans les registres anciens et nouveaux (2,4\% par an) (Figure 3 ). Par contre l'augmentation est plus importante dans les registres nouveaux (3,0\% par an) (Figure 4) que dans les anciens ( $1,8 \%$ par an) en ce qui concerne les non séminomes. Pour la France les séminomes sont passés de 1,50 en 1978 à 2,46 pour 100000 en 2000 (soit une augmentation de $2,4 \%$ par an) et les non séminomes de 1,18 à 1,95 sur la même période (soit une augmentation également de $2,4 \%$ par an).

Les séminomes sont en augmentation quasi constante avec la cohorte de naissance (Figure 5). Ceci n'est pas vrai des non séminomes pour lesquels le risque a diminué pour les hommes nés entre les deux guerres mondiales. À partir des années 40 , séminomes et non séminomes évoluent de la même façon.

\section{Estimation en l'an 2000.}

Les taux de mortalité et d'incidence par âge estimés pour l'an 2000 sont donnés au Tableau 1. On peut estimer qu'actuellement il y a 1500 nouveaux cas de cancers du testicule chaque année en France, dont 960 pour des hommes de 15 à 40 ans.

\section{DISCUSSION}

Comme dans la plupart des pays, la mortalité par cancer du testicule est en diminution. Ceci est dû principalement à l'introduction des chimiothérapies au cisplatine au milieu des années 70 et à l'optimisation des chimiothérapies et de la radiothérapie depuis cette période. Ces améliorations ont permis de traiter efficacement même les formes métastatiques.

L'incidence française, donnée au Tableau 1, ne doit pas cacher que les taux d'incidence sont très différents d'un département à l'autre. Il existe un gradient nord-sud et estouest. Les taux les plus hauts se trouvant dans le nord-est (Bas-Rhin : 8,3 pour 100000 , standardisé sur le monde) et les plus bas dans le sud-ouest (Hérault : 3,2 pour 100000, standardisé sur le monde). Les taux estimés pour 2000, standardisés sur la population mondiale, dans les différents registres illustrent bien ce propos (Tableau 2). Les taux vont du simple au double et le Bas-Rhin approche des taux les plus élevés que l'on rencontre dans les pays nordiques, en particulier au Danemark où le taux atteint 9,2 pour 100000 , ou encore la Suisse où les taux varient entre 7,3 et 10,3 [9].

Si l'incidence des séminomes n'a cessé d'augmenter avec la cohorte de naissance, il n'en est pas de même pour les non séminomes où l'on observe une diminution pour les cohortes de naissance entre les deux guerres mondiales. Ensuite l'évolution se superpose à celle des séminomes à partir de la cohorte de naissance 1940. Bien que nous ne puissions exclure un artefact, l'analyse par cohorte et par âge confirme les risques relatifs de la Figure 5. La prise en compte d'autres formes histologiques parmi les non séminomes ne semble pas être une explication valable. Cela produirait une augmentation et non pas une diminution, en effet c'est pour les cohortes les plus anciennes que la diminution est observée, l'augmentation étant dans les cohortes les plus récentes pour lesquelles le diagnostic a été fait récemment c'est-à-dire à une période où le diagnostic histologique ne pose plus de problème et où les dossiers médicaux permettent aux registres de coder correctement le type histologique. Une possibilité pourrait être un sous-enregistrement des cancers du testicule chez les personnes âgés, mais on ne voit pas pourquoi cela toucherait plus spécifiquement les non séminomes. Une troisième hypothèse pourrait être le problème des tumeurs mixtes pour lesquelles les règles de classification n'étaient pas claires au début de la période d'enregistrement. Néanmoins ces tumeurs ont toujours été classées avec le type de plus mauvais pronostic et c'est cette règle qui a été utilisée dans cette analyse sur l'ensemble de la période d'analyse. Le fait que pour ces cohortes les estimations reposent plutôt sur des hommes âgés d'au moins 40 ans, et donc là où l'incidence est faible et plus instable, ne semble pas non plus être une explication car la représentation par cohorte en fonction de 




Figure 1 : Évolution de la mortalité par cancer du testicule en France.

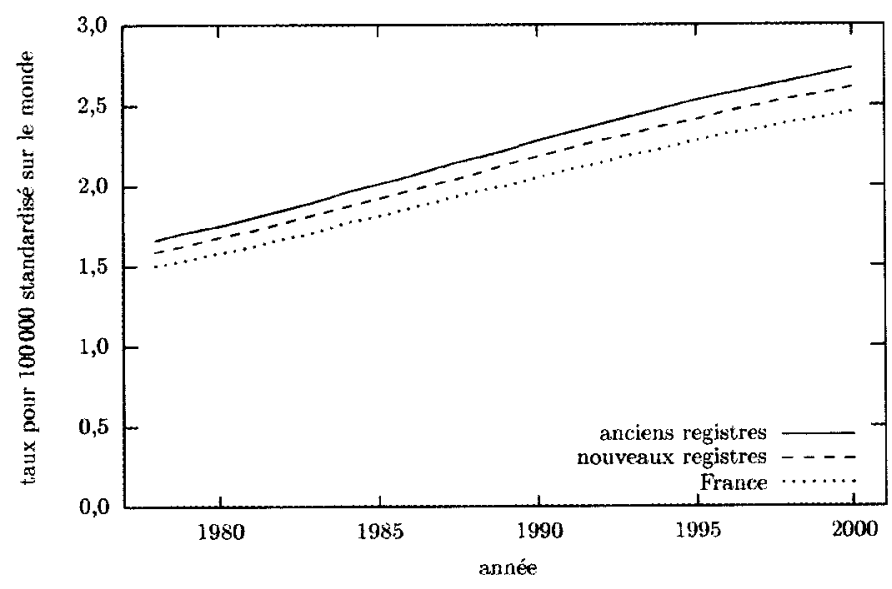

Figure 3 : Évolution de l'incidence des séminomes en France.



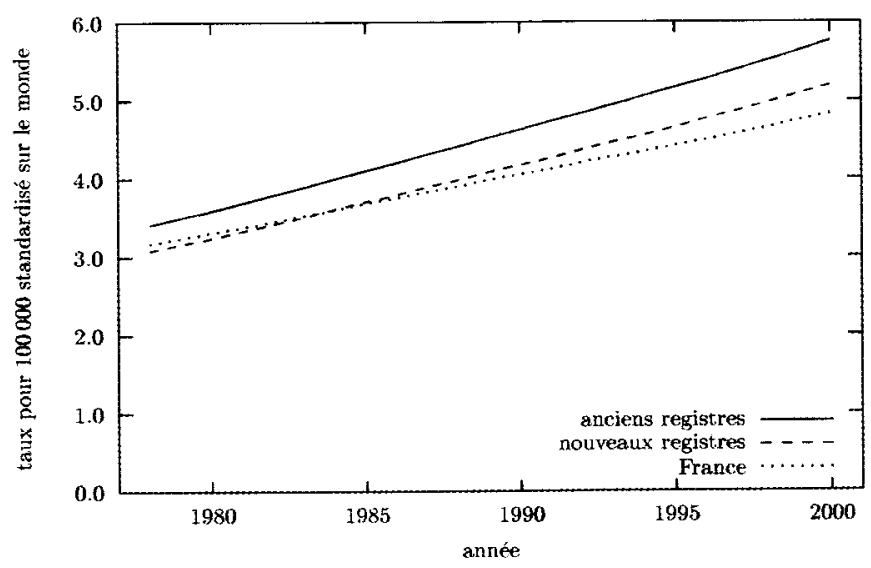

Figure 2: Évolution de l'incidence du cancer du testicule en France.

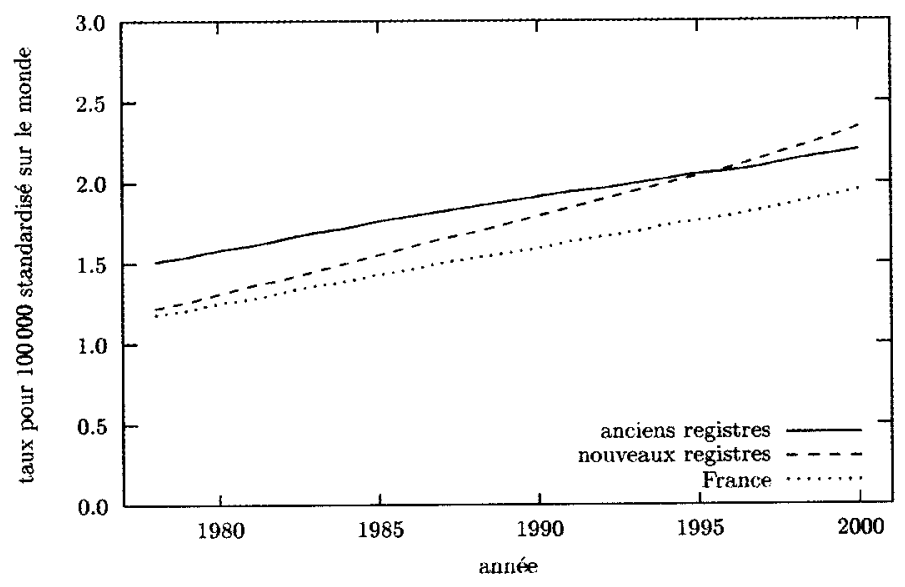

Figure 4 : Évolution de l'incidence des non séminomes en France.
Figure 5 : Évolution de l'incidence par type histologique (risques relatifs). 
Tableau 1 : Taux de mortalité et d'incidence pour 100000 par âge en France en 2000.

\begin{tabular}{|c|c|c|c|c|}
\hline \multirow[t]{2}{*}{$\overline{\text { age }}$} & \multirow[t]{2}{*}{ mortalité } & \multicolumn{3}{|c|}{ incidence } \\
\hline & & tous types & séminomes & non séminomes \\
\hline$[00 ; 04]$ & 0,00 & 0,66 & 0,00 & 0,54 \\
\hline$[05 ; 09]$ & 0,00 & 0,11 & 0,00 & 0,11 \\
\hline$[10 ; 14]$ & 0,00 & 0,15 & 0,00 & 0,10 \\
\hline$[15 ; 19]$ & 0,08 & 2,36 & 0,20 & 1,75 \\
\hline$[20 ; 24]$ & 0,31 & 6,98 & 1,21 & 4,72 \\
\hline$[25 ; 29]$ & 0,48 & 11,28 & 3,95 & 6,15 \\
\hline$[30 ; 34]$ & 0,47 & 13,44 & 7,31 & 5,36 \\
\hline$[35 ; 39]$ & 0,42 & 12,33 & 8,20 & 3,62 \\
\hline$[40 ; 44]$ & 0,36 & 8,72 & 6,39 & 2,00 \\
\hline$[45 ; 49]$ & 0,35 & 6,63 & 5,03 & 1,26 \\
\hline$[50 ; 54]$ & 0,25 & 4,64 & 3,54 & 0,81 \\
\hline$[55 ; 59]$ & 0.28 & 3,57 & 2,64 & 0,64 \\
\hline$[60 ; 64]$ & 0,28 & 2,34 & 1,64 & 0,39 \\
\hline$[65 ; 69]$ & 0,35 & 1,70 & 1,13 & 0,24 \\
\hline$[70 ; 74]$ & 0,45 & 1,39 & 0,93 & 0,19 \\
\hline$[75 ; 79]$ & 0,74 & 0.83 & 0,47 & 0,12 \\
\hline$[80 ; 84]$ & 0,93 & 1,30 & 0,78 & 0,26 \\
\hline$[85:++]$ & 2,15 & 1,71 & 0,85 & 0,28 \\
\hline
\end{tabular}

Tableau 2 : Taux d'incidence pour 100000 standardisés sur la population mondiale dans les registres français en 2000.

\begin{tabular}{lc}
\hline Registre & taux pour 10000 standardisés sur le monde \\
\hline Hérault & 3,2 \\
Calvados & 3,8 \\
Somme & 4,0 \\
Tarn & 4,3 \\
Manche & 4,3 \\
Doubs & 4,3 \\
Isère & 4,7 \\
Haut-Rhin & 7,7 \\
Bas-Rhin & 8,3 \\
\hline
\end{tabular}


l'âge est cohérent avec un effet quel que soit l'âge : on retrouve le même ordre sur les cohortes à âge fixé. Møller [8] a noté au Danemark une baisse de l'incidence de l'ensemble des tumeurs germinales du testicule pour les cohortes de naissance autour de la deuxième guerre mondiale. Ceci a également été observé par Bergström et al. [2] en Norvège et en Suède. Møller en avait déduit que c'était dû à des facteurs de risque qui devaient agir très tôt dans la vie et probablement in utero.

L'augmentation du nombre de cancers du testicule chez l'homme jeune pose la question de la prise en charge extramédicale de ces personnes, tant sur le plan psychologique que sur les possibilités de procréation après le traitement. Ceci incite à développer encore plus les réflexions et les partenariats entre les services traitants et les autres structures telles que les CECOS.

\section{CONCLUSION}

Les évolutions de la mortalité et de l'incidence du cancer du testicule en France sont conformes à ce qui est observé dans la majorité des pays : une diminution de la mortalité et une augmentation de l'incidence. En France, on peut estimer à 1500 le nombre de nouveaux cas en l'an 2000, dont 960 entre 15 et 40 ans. L'évolution différente des non séminomes entre les deux guerres mondiales reste à expliquer.

\section{REFERENCES}

1. AALEN O.O., TRETLI S. : Analyzing incidence of testis cancer by means of a frailty model. Cancer Causes Control, 1999, 10 : 285-292.

2. BERGSTRÖM R., ADAMI H.O., MÖHNER M. et al. : Increase in testicular cancer incidence in six european countries : a birth cohort phenomenon. J. Natl. Cancer Inst., 1996, 88 : 727-733.

3. COLONNA M., EXBRAYAT C., LAUNOY G. et al. : Incidence du cancer en France : Estimations régionales 1985 - 1995, Francim, Paris, 1999.

4. ESTEVE J., BENHAMOU E., RAYMOND L. : Méthodes statistiques en épidémiologie descriptive. INSERM, Paris, 1993.

5. HUYGHE E., THONNEAU P.F. : Augmentation de l'incidence du cancer du testicule : état de la question. Andrologie, 2000, 10: 302-311.

6. JOUANNET P., AUGER I. : Is the incidence of testicular cancer on the increase in France ? Presse Med., 1995, 24 : 1133.

7. LUTZ J.M., MENEGOZ F. : Épidémiologie des cancers du testicule. Ann. Urol., 1992, $26:$ 301-305.

8. MØLLER H. : Clues to the atiology of testicular germ cell tumors from descriptive epidemiology. Eur. Urol., 1993, 23 : 815.

9. PARKIN D.M., WHELAN S.L., FERLAY J., RAYMOND L., YOUNG $\mathrm{J}$. (eds) : Cancer incidence in five continents. IARC Scientific publications, volume VII. IARC, Lyon, 1997.
10. ZHENG T., HOLFORD T.R., MA Z., WARD B.A., FLANNERY J., BOYLE P. : Continuing increase in incidence of germ-cell testis cancer in young adults : experience from Connecticut, USA, 1935-1992. Int. J. Cancer, 1996, 65 : 723-729.

\section{ABSTRACT}

Testis cancer trends in France

\section{GuY HEDELIN, Laurent REMONTET}

As in many countries, the mortality rate of testicular cancer in France has decreased from 0.75 in 1978 to 0.25 per 100,000 in 2000 (standardized on world population).

Over the same period, the incidence rate increased from 3.17 in 1978 to 4.82 per 100,000 in 2000 (standardized on world population). However, although the incidence of seminoma has increased continually with all birth cohorts, the incidence of non-seminomas first decreased for the men born between the first and second world wars and then increased at the same rate as the seminoma rate. No explanation for this pattern has yet been provided, but it does not appear to be simply an artefact.

A North/South and East/West gradient has been observed, as the incidence varies from 4.0 in the South West to 8.0 in the North East.

There is an estimated 1,500 new cases of testicular cancer each year in France, including 960 new cases for men between the ages of 15 and 40 .

Key words: testicular cancer, incidence, mortality, trend, epidemiology 